\title{
Beitrag zur kongenitalen Dystopie der Niere. $\left.{ }^{1}\right)$ (Beckenniere.)
}

\author{
Von \\ Dr. R. Bretschneider \\ aus Leipzig.
}

M. H.! Ich bin auf das angekündigte Thema gekommen durch einen Fall, den vor kurzer Zeit zu operieren ich Gelegenheit hatte, und da die Fälle von sogenannten Beckennieren nicht so ganz selten zu sein scheinen und ausserdem schon vielfach zu sehr verhängnisvollen therapeutiscben Eingriffen geíührt haben, will ich Ihnen den Fall nicht vorenthalten. Die Krankengeschichte des von mir operierten Falles ist folgende:

Es handelt sich um eine 30jährige Patientin, die vor etwa 3 Jahren einen langdanernden, sehr schweren Partus (mittels Forzeps) durchgemacht hat. Der damalige Hausarzt hat, wie er mir mitteilt, von einem Tumor nichts feststellen können, sondern als Ursache für den schweren Forzeps ein Missverhältnis zwischen Kopf und Becken angenommen. Die Pat. hat das Wochenbett ganz gut überstanden und auch in der Folgezeit sich ganz wohl gefühlt. Ab und zu sind später in der linken Seite plötzlich Leibschmerzen kolikartiger Natur aufgetreten, die sie nicht weiter beachtete und für Blähungsbeschwerden hielt, da sie bald wieder vergingen. Im Oktober und November vorigen Jahres setzte die sonst ganz regelmässige Periode aus; dann traten Blutungen und Leibschmerzen ein, und der hinzugezogene Hausarzt machte eine Kurettage in der Meinung, es sei ein Abort. Da er aber nur sehr wenig entfernen konnte, und da die nach einiger Zeit wegen Leibschmerzen (kolikartig) vorgenommene bimanuelle Untersuchung einen Tumor links neben dem Uterus von ungefähr Apfelgrösse ergab und somit an die Möglichkeit einer Extrauteringravidität gedacht werden musste, so schickte er die Pat. in meine Klinik behufs Vornahme einer Operation. Die Aufnahme in die Klinik erfolgte am 28. XII. 11. Die Untersuchung meinerseits ergab, dass livide Verfärbung der Vagina, Auflockerung derselben und Kolostrum in den Brüsten nachzuweisen und links vom Uterus, mehr der Beckenwand anliegend, aber mit dem Uterus deutlich in Zusammenhang stehend ein etwa apfelgrosser Tumor von etwas weicher, teigiger Konsistenz festzustellen war. Derselbe schien mir, da er sich von der

1) Nach einem in der Gesellsch. für Geburtsh. u. Gyn. zu Leipzig am 19. Febr. 1912 gehaltenen Vortrag. 
Beckenwand nicht ganz abtasten liess, entweder etwas verwachsen oder intraligamentär entwickelt $z u$ sein. Mit Rücksicht auf die Anamnese und die vorhandenen Graviditätszeichen dachte ich auch in erster Linie an einen extrauterinen Fruchtsack, in zweiter Linie aber auch an eine intraligamentär entwickelte Cyste. Da eine strikte Indikation zur Operation zunächst nicht vorlag und, falls es eine Extrauteringravidität war, man mit der Möglichkeit rechnen konnte, dass es keine wachsende, sondern eine in Resorption begriffene sei, so entschloss ich mich, die Pat. zunächst etwas zu beobachten. Da am nächsten Tage, wie Sie sich durch Einsicht in die mitgebrachte Kurve überzeugen können, eine auffallende Pulssteigerung auftrat und zugleich das Allgemeinbefinden gestört wurde, sowie wieder kolikartige Schmerzen in der linken Leibseite auftraten, glaubte ich, es bereite sich eine Katastrophe vor (Platzen des vermeintiichen Fruchtsackes), und um dem zuvorzukommen, entschloss ich mich zur Laparotomie. Dieselbe wurde am.30. XII, ausgeführt und ergab folgendes Resultat: Uterus ohne Besonderheiten, desgleichen die rechten Adnexe. Die linke Tube war ebenfalls schlank, aber mit dem abdominalen Ende und zugleich mit dem etwas cystisch degenerierten Ovarium mit jenem schon durch die bimanuelle Untersuchung festgestellten Tumor verwachsen. Diese Verwachsungen liessen sich leicht trennen, und nun zeigte es sich, dass dieser Tumor extraperitoneal lateral vom Rektum, etwa in der Gegend der Incis. ischiadica sin. lag. Er liess sich auf der Unterlage leidlich gut hin und her verschieben, hatte teigige, fast ballotierende Konsistenz und eine etwas höckerige Oberfläche. Um die Natur des Tumors etwas genauer festzustellen, spaltete ich das Peritoneum über demselben in sagittaler Richtung und versuchte vorsichtig, den Tumor stumpf mit dem Finger aus seinem Bett zu schälen und etwas emporzuheben. Hierbei gab es plötzlich eine derartig abundante Blutung, dass das ganze kleine Becken mit Blut überschwemmt wurde und jedes weitere Operieren unmöglich machte.

Ich glaubte, die Vena hypog. angerissen zu haben, was aber nicht der Fall war. Durch Kompression der Aorta gelang es, die blutende Stelle zu finden. Es war ein Gefässbündel am medialen Blatt des abgelösten Peritoneums (nach dem Rektum zu), das unzweifelhaft der Gefässstiel des Tumors sein musste. Die weitere Exstirpation des Tumors war damit so gut wie beendet. Die genanere Besichtigung desselben ergab, dass es sich um eine Niere, also um eine sogenannte Beckenniere handelte. Diẻ sofort vorgenommene weitere Inspektion des Abdomens ergab, dass die linke Niere an der normalen Stelle fehlte, die rechte Niere in normaler Grösse unter der Leber zu palpieren war. Das abgerissene Ureterende wurde bald gefunden und versorgt, die Wundhöhle durch den Douglas drainirt und die Bauchhöhle geschlossen.

Die exstirpierte Niere habe ich Ihnen mitgebracht. Sie zeigt nicht die gewöhnliche gebogene Gestalt, sondern die etwa einer platt gedrückten Birne; ausserdem ist bemerkenswert, dass das Nierenbecken nicht wie gewöhnlich am Hilus, sondern vollständig auf einer Breitseite aufliegt, nirgends den Rand überragt und deutlich erweitert ist (beginnende Hydronephrose). - Die Rekonvaleszenz gestaltete sich folgendermassen: Wie Sie aus der Kurve ersehen, trat in den folgenden Tagen eine Pulssteigerung bis zn 132 auf, die Temperatur erreichte am 3. Tage mit 38,1 den höchsten Stand und fiel bald zur Norm ab, der Puls hingegen erreichte erst am 10.-12. Tage die Norm wieder. Vom 3.-8. Tagè waren Symptome urämischer Natur zu beobachten, bestehend 
in Kopfschmerz, Brechreiz, grosser Unruhe, Hautjucken und heftigen Durchfällen, die erst vom 9. Tage post operationem wieder schwanden. Das Abdomen war während dieser Zeit nirgends gespannt oder druckempfindlich. Von grösster Wichtigkeit für den weiteren Verlauf war natürlich die Leistungsfähigkeit der rechten Niere. Um diese nicht zu überlasten, wurde anfangs die Flüssigkeitszufuhr so weit herabgesetzt, dass Pat. sozusagen immer unter etwas Durstgefühl stand, dementsprechend wurde natürlich auch die Zufuhr von Eiweissstoffen möglichst eingeschränkt, weniger die Zufuhr von Fetten und Kohlehydraten. Die Urinmengen, die abgesondert wurden, betrugen nach 48 Stunden im ganzen $600 \mathrm{ccm}$, also unverhältnismässig wenig, was mich aber nicht sehr wunderte, da sicher der ziemlich starke Blutverlust bei der Operation und die verringerte Flüssigkeitszufuhr als ursächliche Momente mit in Frage kamen. Vom 3.-7. Tage an konnte der Urin nicht gemessen werden wregen der bestehenden Durchfälle, während welcher Zeit sicher viel Flüssigkeit durch den Darm und noch wenig durch die Niere ausgeschieden wurde. Vom 8. Tage an konnte der Urin wieder in toto aufgefangen werden, es waren $450 \mathrm{ccm}$, von da ab nahm die Harnmenge täglich zu, die Zahl der Darmentleerungen ab, am 25. Tage post oper. (Entlassungstag) wurden $1500 \mathrm{ccm}$, also normale Mengen Urin abgesondert. Die Pat. wurde bei bestem Wohlbefinden entlassen, und die erst vor kurzem erfolgte Nachuntersuchung ergab ein durchaus befriedigendes Resultat.

M. H.! Der vorliegende Fall hat mir Veranlassung gegeben, die Literatur auf diese Rarität hin genauer durehzusehen und daran eigene Schlussfolgerungen zu knüpfen. Zunächst ist zu bemerken, dass die Beckennieren doch nicht so ganz selten sind, als es scheinen mag. Ich finde in der Literatur eine Statistik von Kehrer (1) über 34 Fälle, eine von Sträter (2) über 67 Fälle und eine von Meyer (3) über 14 Fälle; ausserdem sind in den letzen Jahren noch eine Anzahl von Einzelbeobachtungen veröffentlicht worden, so von Menacher (4), Halban (5), Kubinyi (6), Frank (7), Schaad (8), Fedoroff (9), Löwit (10), Lindemann (11), Albrecht (12), Weibl (13) u. a. m. Auch Graser (14), scheint der Ansicht zu sein, dass Beckennieren nicht so ganz selten sind; er fand unter 150 Fällen von dystopen Nieren $15 \mathrm{mal}$ Beckennieren.

Die grössten Schwierigkeiten bereitet naturgemäss immer die Diagnose. Die meisten Fälle von Beckennieren sind erst intra operationem, manche auch erst post operationem als solche erkannt worden, verschiedene wurden ferner entdeckt bei Sektionen als zufälliger Sektionsbefund. Nur bei ganz wenigen Fällen ist es gelungen, die richtige Diagnose ante operationem mit Sicherheit zu stellen. Zur Sicherung der Diagnose sind verschiedene Methoden angegeben worden, von denen nach meiner Ansicht die wichtigste 
der Nachweis der sogenannten renopalpatorischen Albuminurie ist, durch welche es nach Eremitsch (18) in Fedoroff's Klinik in vier Fällen gelang, die Diagnose vor der Operation zu stellen. Auf dieses Phänomen hat seinerzeit auch Menge (19) in einer Sitzung der hiesigen Gesellschaft bei der Wanderniere aufmerksam gemacht, indem er nachwies, dass etwa $1 / 2$ Stunde nach Massage der Niere der Urin eiweisshaltig war. Ferner ist wichtig für die Diagnose die vergleichende Messung der Ureteren und die Röntgenphotographie, worauf Krömer bereits hingewiesen hat und wodurch es auch Müllerheim (20), Albrecht (12) und Kuntzsch (24) gelang, die Diagnose einer Beckenniere zu sichern. Wie ich schon oben andeutete, sind in der bei weitem grössten Mehrzahl der Fälle Fehldiagnosen gestellt worden, meistens wurde ein Ovarialbzw. Adnextumor diagnostiziert, aber auch andere Diagnosen, wie Myom (Schauta), Hämatometra (Kubin yi), perityphlitischer Abscess (bei vereiterter Hydronephrose einer dystropen Niere - Schaad) wurden gestellt. Leider hat der Umstand, dass die Diagnose der Beckenniere so beträchtliche Schwierigkeiten macht, zu recht verhängnisvollen therapeutischen Eingriffen Anlass gegeben. So teilen z. B. Hochenegg (15), Sträter (16), Buss (17) u. a. mit, dass in verschiedenen Fällen der exstirpierte Tumor die einzige Niere war und der Exitus an Anurie erfolgte. Es ist daher mit Rücksicht auf diese trüben Erfahrungen ohne weiteres klar, dass bei allen unklaren Tumoren im kleinen Becken unser therapeutisches bzw. operatives Vorgehen ein ausserordentlich vorsichtiges sein muss. Selbstverständlich werden wir uns hierbei hauptsächlich von der Intensität der Beschwerden und der sonstigen Komplikationen leiten lassen. Man wird sich unschwer zu einem operativen Eingriff entschliessen, wenn die Beckenniere durch Komplikationen, wie Steinbildung, Hydronephrose, Pyelitis usw. erkrankt und zum Teil schon funktionsunfähig ist, und zwar kann hier der operative Eingriff nur in Exstirpation der Beckenniere bestehen. Man wird sich aber auch hier selbstverständlich erst davon überzengen müssen, ob die andere Niere die volle Funktion schon übernommen hat oder übernehmen kann. Nicht so einfach ist die Frage zu entscheiden, was man tun soll, wenn die Beckenniere noch leidlich funktionsfähig oder ganz gesund ist und nur subjektive Beschwerden, wie Kreuzschmerzen, Menstruationsbeschwerden, leichte Koliken, hartnäckige Behinderung der Defäkation (Fall Hochenegg) usw. bestehen, oder wenn sie ernste Geburtshindernisse 
abgegeben haben, wie in meinen und anderen Fällen, oder zu Abort geführt haben, oder wenn man den Tumor erst intra operationem als Beckenniere erkennt. Hier käme ein Verfahren in Frage, das Halban (5) einmal mit Erfolg ausgeführt hat und auf das Fedoroff und Sträter auch schon hingewiesen und auch zum Teil mit Erfolg ausgeführt haben, nämlich die Beckenniere aus dem kleinen Becken in das grosse zu verlagern. Wenn ich meinen Fall hierbei berücksichtige, so wäre diese Operation gescheitert an der Kürze und Unnachgiebigkeit des Gefässstiels und des Ureters; denn beim ersten Versuch, den Tumor aus seinem Bett herauszuheben, gab es infolge Zerreissens der Hauptgefässe jene oben erwähnte abundante Blutung, die zur Exstirpation des Tumors führen musste. Unbedingt warnen möchte ich daher vor diesem Versuch, wenn man die Beckenniere erst während der Operation als solche erkennt und festgestellt hat, dass die andere Niere fehlt oder rudimentär entwickelt ist; die Gefahr der Läsion des Gefässstieles bei der Operation oder der sekundären Nekrose der nach oben verlagerten Niere ist ausserordentlich gross. Ist die andere Niere normal, so kann man wohl den Versuch einer Verlagerung der Niere nach oben machen; er wird aber wohl vielfach scheitern an der Kürze des Ureters und der Blutgefässe, eine Ansicht, der auch Engström (21) beizupflichten scheint. Wenn daher eine gesunde Beckenniere bedeutende Beschwerden gemacht, oder eventuell ein schweres Geburtshindernis abgegeben hat, so wird man meiner Ansicht nach besser daran tun, dieselbe zu exstirpieren, als den Versuch der Verlagerung nach oben zu machen, zumal wenn man bedenkt, dass eine eventuell eintretende Gangrän der nach oben verlagerten Niere nicht immer harmlos abzulaufen braucht; und wenn man weiterhin bedenkt, dass gerade verlagerte Nieren eher zu krankhaften Prozessen wie Hydronephrose, Steinbildung, chronische Pyelitis usw. zu neigen scheinen als normal gelegene. Ist die Beckenniere schon irgendwie pathologisch verändert, so ist dieselbe wohl unter allen Umständen zu exstirpieren, vorausgesetzt natürlich, dass die andere Niere sich als völlig funktionsfähig erwiesen hat. Fehlt die andere Niere, so ist auch die kranke Beckenniere in situ zu lassen und die Bauchhöhle zu schliessen.

Was nun die Gefässversorgung der kongenital dystopen Nieren anbetrifft, so konnte ich in meinem Falle feststellen, dass nur eine und zwar ziemlich kräftige Arterie vorhanden war und dass die- 
selbe aus einer Hämorrhoidalis (anscheinend der Superior) abging. Ich will es hierbei nicht unterlassen, auf die sehr ausführliche und interessante Arbeit von 0. Meyer in Ziegler's Beiträgen hinzuweisen. Es geht aus dieser hervor, dass eine gewisse Gesetzmässigkeit in dem Abgang der renalen Gefässe von den grösseren Arterien nicht. besteht, nur das scheint sicher zu sein, dass die Gefässversorgung der kongenital dystopen Nieren häufig eine mehrfache zu sein scheint, ein Umstand, der naturgemäss das operative Vorgehen, namentlich die Verlagerung nach oben, erschweren muss.

Am fatalsten für den Operateur und verhängnisvollsten für die Patientin ist die Situation natürlich dann, wenn, wie ich schon oben erwähnte, der Tumor erst nach der Exstirpation als Niere erkannt. und zugleich das Feblen der anderen Niere festgestellt wird. Das einzig mögliche Verfahren, was man hierbei noch zur Rettung der Patientin einschlagen könnte, wäre die Reimplantation der Niere. Die Carellschen Versuche (22) am Tierexperiment lassen diesen Versuch jedenfalls zunächst als nicht ganz aussichtslos erscheinen. Carell exstirpierte bekanntlich bei 6 Hunden beide Nieren und implantierte mittels Gefäss- und Ureternaht wieder eine Niere; 5 Hunde überstanden die Operation, 4 gingen später an Ureterstenose, Pyelitis usw. zugrunde, einer scheint jedoch die Operation. dauernd überstanden zu haben. Unger (23) hat die gleichen Tierexperimente ebenfalls ausgeführt, anscheinend mit nicht so günstigen Erfolgen, aber er scheint doch auch anzunebmen, dass diese Versuche nicht ganz aussichtslos sind nach einem Fall, den er in der Berliner medizinischen Gesellschaft am 2. März 1910 vortrug. In diesem Falle hatte er bei einem Mädchen mit schwerster Nephritis eine Affenniere mitsamt der Vena cava und Aorta in die durchschnittene Vena bzw. Arteria femoralis eingepflanzt. Der Exitus erfolgte 32 Stunden p. op., die Gefässe seien aber nicht thrombosiert gewesen und die Niere hätte einen lebensfrischen Eindruck gemacht. Dennoch erscheint es mir trotz dieser nicht ganz ungünstigen Experimente recht fraglich, ob diese Technik sich am Menschen jemals soweit ausbilden lässt, dass eine Reimplantation einer exstirpierten Niere einige Aussicht auf dauernde Heilung darbieten wird. Immerhin wird es für einen Operateur, der in der unglücklichen Lage ist, die Beckenniere als einzige Niere entfernt zu haben, ein kleiner Trost sein, wenn er diesen letzten Rettungsweg noch einschlagen kann oder zum wenigsten nicht unversucht gelassen hat. 
I. H.! Wenn ich meine Erfahrung mit dem vorliegenden Falle und die Ergebnisse der hauptsächlichsten Literaturangaben einer kritischen Betrachtung unterziehe, so komme ich, wenn ich es nochmals kurz zusammenfassen darf, zu folgenden Schlussfolgerungen:

1. Bei jedem retroperitoneal gelegenen Beckentumor muss man an das Vorhandensein einer Beckenniere denken.

2. Bei jedem Adnextumor unklarer Natur sind zur Sicherung der Diagnose das Phänomen der renopalpatorischen Albuminurie zu prüfen, vergleichende Messungen der Ureteren vorzunehmen und Röntgenphotographien mit im Ureter liegendem Katheter mit Metallmandrin anzufertigen.

3. Wird der Tumor erst intra operat. als Beckenniere erkannt und dieselbe als normal befunden, so ist ein Versuch, dieselbe ins grosse Becken zu verlagern, nur dann vorzunehmen, wenn die andere Niere als vollauf funktionsfähig befunden wird. Dieser Versuch wäre gerechtfertigt, wenn die Beckenniere als eventuelles Geburtshindernis in Frage käme; er ist unter allen Umständen zu unterlassen bei Mangel oder rudimentärer Entwicklung der anderen Niere, da er leicht zur Exstirpation führen kann. Die Laparotomie wäre dann als nur zu diagnostischen Zwecken vorgenommen zu betrachten.

4. Die Beckenniere ist za exstirpieren, wenn |sie sehr starke Beschwerden macht oder schon pathologisch verändert ist. Ist sie jedoch die einzige Niere, so ist sie unter keinen Umständen operativ anzugreifen, auch wenn sie Beschwerden macht oder nicht mehr als ganz gesund erscheint.

\section{Literatur.}

1. Kehrer, Chrobak-Festschrift 1903.

2. Sträter, Zentralbl. f. Gyn. 1907. No. 7 .

3. Meyer, Ziegler's Beiträge zur allgem. pathol. Anat. u. z: allgem. Pathol. Bd. 44. H. 1.

4. Menacher, Zentralbl. f. Patbol. 1909.

5. Halban, Wiener klin. Wochenschr. 1910. No.4.

6. Kubinyi, Zentralbl. f. Gyn. 1910. No. 8.

7. Frank, Fortschr. d. Geburtsh. u. Gyn. 1909. S. 352.

8. Schaad, Zentralbl. f. allg. Path. u. path. Anat. 1909.

9. Fedor off, Zeitschr. f. Urol. Bd. 3.

10. Löwit, Zeitschr. f. gyn. Urol. Bd. 1. H. 3. 
322 Bretschneider, Beitrag zur kongenitalen Dystopie der Niere.

11. Lindemann, Münch. med. Wochenschr. 1911. S. 2180.

12. Albrecht, Münch. med. Wochenschr. 1911. S. 2689.

13. Weibl, Wiener klin. Wochenschr. 1908. No. 47.

14. Graser, Deutsches Arch. f. klin. Med. Bd. 55.

15. Hochenegg, Wiener klin. Wochenschr. 1900. No. 1.

16. Sträter, Zentralbl. f. Gyn. 1905. No. 42.

17. Buss, Zeitschr. f. klin. Med. Bd. 38.

18. Eremitsch, Zentralbl. f. Gyn. 1910, No. 4.

19. Menge, Verhandlungen der Ges. f. Geb. u. Gyn. in Leipzig. 1900.

20. Müllerheim, Ges. f. Geb. u. Gyn. zu Berlin. Sitzg. v. 22. April 1904

21. Engström, Münch, med. Woohenschr. 1903. S. 1039.

22. Carrel, Münch. med. Wochenschr. 1909. S. 356.

23. Unger, Münch. med. Wochenschr. 1910. S. 556.

24. Kuntzsch, Zentralbl. f. Gyn. 1911. S. 1061. 